\title{
Spectral Efficiency Comparison of OFDM and MC-CDMA with Carrier Frequency Offset
}

\author{
Junaid AHMED \\ Dept. of Electrical Engineering, COMSATS Institute of Information Technology, Tarlai Kalan, 45550 Islamabad, Pakistan \\ junaid@comsats.edu.pk
}

Submitted March 4, 2016 / Accepted December 15, 2016

\begin{abstract}
Inter-carrier interference and multiple access interference due to carrier frequency offset $(C F O)$ are two major factors that deteriorate the performance of orthogonal frequency division multiple access (OFDMA) and multicarrier code division multiple access (MC-CDMA) in wireless communication. This paper presents a new mathematical analysis for spectral efficiency of OFDMA communication systems over a frequency selective Rayleigh fading environment in the presence of multiple users. It also compares the spectral efficiency performance of OFDMA and MC-CDMA at different load, signal-to-noise ratio, CFO and delay spread conditions. MC-CDMA is found to be more resilient to CFO in general, however, OFDMA performs better at high load.
\end{abstract}

\section{Keywords}

OFDM, MC-CDMA, spectral efficiency, carrier frequency offset, inter-carrier interference, multiple access interference

\section{Introduction}

Orthogonal frequency division multiple access (OFDMA) is a well known technique used in both wireless and wired communication applications. It offers high data rate, high spectral efficiency and multiple access capability. It is used in standardized wireless communication techniques like long term evolution (LTE), WiMax, digital video broadcasting, etc. Multicarrier code division multiple access (MC-CDMA) is a promising future communication technique [1]. It combines OFDM and code division multiple access (CDMA) to give advantages of both, including high data rate, high spectral efficiency, robustness to frequency selective fading, multiple access capability and narrow band interference rejection among others [2-4]. However, due to carrier frequency offset (CFO) both OFDMA and MCCDMA systems suffer from inter-carrier interference (ICI) and multiple access interference (MAI), [5-9]. The CFO appears due to frequency difference of local oscillators in transmitter and receiver, and the Doppler shift in frequency due to motion of transmitter and/or receiver. The spectral ef- ficiency degradation of MC-CDMA, in the presence of CFO, over a frequency selective Rayleigh fading environment is studied in [10], [11]; a lower bound of spectral efficiency in the presence of asynchronous interferers is derived. Previous research work on the performance of OFDMA in the presence of CFO has mostly focused on symbol/bit error rate [12-16]. The analyses mostly use Gaussian or improved Gaussian approximation, which are not accurate due to correlation in frequency response of the adjacent subchannels, especially at low bit error rates [17]. In [18-20], average capacity/ spectral efficiency of OFDM with CFO in Rician and Rayleigh fading environment is analyzed respectively. However, their analysis can not be extended to multiuser OFDMA that considers interference from other users (though in [19] accurate conditional Gaussian approximation is used). The presence of multiple users, which act as interferers, is worth considering because CFO results in MAI to other users. Each transmitter has different $\mathrm{CFO}$ as compared to the receiver, therefore, CFO mitigation techniques are unable to cater for all the different CFO's due to transmitter heterogeneity.

In this paper, we derive a new expression for the lower bound of spectral efficiency of an asynchronous uplink OFDMA system that takes into account degradation due to $\mathrm{CFO}$ in the presence of multiple transmitters. We use this expression to compare the spectral efficiency of OFDMA with that of MC-CDMA. We base our analysis on accurate conditional Gaussian approximation for ICI and MAI, and consider the fading experienced by subcarriers to be correlated. The complexity of the numerical calculations of our result depends on the number of subcarriers, and does not increase with an increase in the number of transmitters. In Sec. 2, we present the system model, and in Sec. 3, we analyze the spectral efficiency. Numerical results are given in Sec. 4 , and Sec. 5 concludes the paper.

\section{System Model}

Consider an asynchronous OFDMA uplink mobile communication system with $K$ transmitters in a small geographical area. Let the transmitted signal of the $k^{\text {th }}$ transmitter, be represented during an arbitrary signaling interval as 


$$
s_{k}(t)=\sum_{n=0}^{N_{c}-1} \sqrt{E_{n, k}} b_{n, k} \mathrm{e}^{\mathrm{j} 2 \pi f_{n, k} t}, \quad t \in\left[-T_{g}, T\right] .
$$

We consider $k=1$ as the desired transmitter and the rest $(k>1)$ as other users or interferers. In (1), $b_{n, k}$ are the modulated symbols; these are assumed to be complex valued, independent and identically distributed random variables with unit variance. $E_{n, k}$ is the energy assigned to subcarrier $n$, where $n=0,1,2, \ldots, N_{c}-1$. We assume channel state information (CSI) is only available at the receiver and not at the transmitter, hence, the total energy is the same for each user and uniformly distributed over $N_{c}$ subcarriers, $E_{0, k}=E_{1, k}=\ldots=E_{n, k}=E$. We also assume that all transmitters use the same number of subcarriers. $f_{n, k}$ is frequency of the $n^{\text {th }}$ subcarrier, used by $k^{\text {th }}$ transmitter. All subcarriers are assumed to be equally spaced in frequency, giving $f_{n, k}-f_{m, k}=(n-m) / T$, where $T$ is the symbol duration and $T_{g}$ is the length of cyclic prefix. The transmitted signal is subjected to a frequency-selective multipath Rayleigh fading channel with a transfer function, $G_{n, k}=\sum_{l=0}^{L-1} g_{l, k} \mathrm{e}^{-\mathrm{j} 2 \pi f_{n} \tau_{l, k}}$, where $g_{l, k}$ and $\tau_{l, k}$ are complex amplitude and propagation delay of the $l^{\text {th }}$ path, respectively, and $\tau_{0, k} \leq \tau_{1, k} \leq \ldots \leq \tau_{L-1, k}$. We assume that all $g_{l, k}$ are zero mean uncorrelated complex Gaussian random variables with normalized power, such that, $\sum_{l=0}^{L-1} \mathbb{E}\left[\left|g_{l, k}\right|^{2}\right]=1$. Therefore, $\left\{G_{0, k}, \ldots, G_{N_{p}-1, k}\right\}$ are jointly complex Gaussian random variables with non-zero cross correlation. We adopt Jakes' model [21] and the assumptions therein to calculate the cross correlation, which is given as

$$
\mathbb{E}\left[G_{i, k} G_{j, k}^{*}\right]=\frac{1}{1+\mathrm{j} 2 \pi \sigma\left(f_{i}-f_{j}\right)}
$$

where $\sigma$ represents channel delay spread and Doppler frequency has been assumed to be zero.

We assume that cyclic prefix duration is selected such that, $T_{g}>\tau_{L-1, k}$, hence intersymbol interference is completely avoided. Perfect time synchronization is also assumed between the transmitter-receiver pair. Let $r(t)$ be the received signal, after removing the cyclic prefix, it can be represented as

$$
r(t)=\sum_{k=1}^{K} \sum_{n=0}^{N_{c}-1} \sqrt{E} G_{n, k} b_{n, k} \mathrm{e}^{\mathrm{j} 2 \pi f_{n, k} t} \mathrm{e}^{\mathrm{j} 2 \pi \frac{\Delta_{k}}{T} t}+\eta(t)
$$

where $\eta(t)$ is additive white Gaussian noise (AWGN) with two sided power spectral density of $N_{0} / 2$. The CFO of $k$-th transmitter and the receiver is $\Delta_{k} / T$, where $\Delta_{k}$ represents CFO normalized to the frequency separation between adjacent subcarriers.

The received signal is decoded by a conventional correlation receiver. Let $Z$ represent the receiver decision variable for the $n=1$ subcarrier of the desired $(k=1)$ user, then

$$
\begin{array}{r}
Z=\frac{1}{T} \int_{0}^{T} G_{1,1}^{*} \mathrm{e}^{-\mathrm{j} 2 \pi f_{1,1} t}\left(\sum_{k=1}^{K} \sum_{n_{k}=0}^{N_{c}-1} \sqrt{E} G_{n, k} b_{n, k} \mathrm{e}^{\mathrm{j} 2 \pi f_{n, k} t}\right. \\
\left.\times \mathrm{e}^{\mathrm{j} 2 \pi \frac{\Delta k}{T} t}+\eta(t)\right) .
\end{array}
$$

The decision variable can be separated into four distinct components $Z=S+I_{1}+I_{2}+N$, where $S, I_{1}, I_{2}$ and $N$ represent, respectively, desired signal, MAI, ICI and AWGN component. These are given respectively as follows:

$$
\begin{gathered}
S=\sqrt{E} b_{1,1} \frac{1}{T} \int_{0}^{T}\left|G_{1,1}\right|^{2} \mathrm{e}^{\mathrm{j} 2 \pi \frac{\Delta_{1}}{T} t} \mathrm{~d} t, \\
I_{1}=\frac{\sqrt{E}}{T} \int_{0}^{T} G_{1,1}^{*} \mathrm{e}^{-\mathrm{j} 2 \pi f_{1,1} t}\left(\sum_{k=2}^{K} \sum_{n=0}^{N_{c}-1} G_{n, k} b_{n, k} \mathrm{e}^{\mathrm{j} 2 \pi f_{n, k} t} \mathrm{e}^{\mathrm{j} 2 \pi \frac{\Delta_{k}}{T} t}\right), \\
I_{2}=\frac{\sqrt{E}}{T} \int_{0}^{T} G_{1,1}^{*} \mathrm{e}^{-\mathrm{j} 2 \pi f_{1,1} t}\left(\sum_{n=0, n \neq 1}^{N_{c}-1} G_{n, 1} b_{n, 1} \mathrm{e}^{\mathrm{j} 2 \pi f_{n, 1} t} \mathrm{e}^{\mathrm{j} 2 \pi \frac{\Delta_{1}}{T} t}\right)^{(5)}, \\
N=\frac{1}{T} \int_{0}^{T} G_{1,1}^{*} \mathrm{e}^{-\mathrm{j} 2 \pi f_{1,1} t} \eta(t) .
\end{gathered}
$$

It is worth mentioning that $I_{2}$ is due to $\Delta_{1}$ only, and it goes to zero when $\Delta_{1}$ is zero, where $\Delta_{1}$ is the CFO of desired transmitter with the receiver. $\Delta_{1}$ is a deterministic quantity that can be estimated. For simplicity, we replace $\Delta_{1}$ with just $\Delta$ in the rest of the paper. As far as the statistics of MAI in (5) and ICI in (6) are concerned, these are large sums of correlated random variables and due to correlation the central limit theorem is not applicable. Hence, the assumption of $I_{1}$ and $I_{2}$ being Gaussian random variables can not be justified. However, if we condition on $G_{0,1}, \ldots, G_{N_{p}-1,1}, \ldots, G_{0, K}, \ldots, G_{N_{p}-1, K}$ then the central limit theorem is applicable as $b_{n, k}$ and $b_{n, 1}$ are independent, identically distributed random variables. We, therefore, approximate $Z$ as a conditional Gaussian random variable, conditioned on $G_{0,1}, \ldots, G_{N_{p}-1, K}$. The conditional variance is given in (12), shown in Appendix, conditional mean is given as follows:

$$
\mathbb{E}\left[Z \mid G_{0,1}, \ldots, G_{N_{p}-1, K}\right]=\sqrt{\frac{E}{T}}\left|G_{1,1}\right|^{2} \operatorname{sinc}\left(\pi \triangle_{1}\right) .
$$

The instantaneous SINR is therefore a random variable given in (13), shown in Appendix, which can be simplified as:

$$
\begin{aligned}
& \operatorname{SINR}=\left|G_{1,1}\right|^{2} / \\
& {\left[\sum_{k=2}^{K} \sum_{n=0}^{N_{c}-1}\left|G_{n, k}\right|^{2} \rho_{n, k}+\sum_{n=0, n \neq 1}^{N_{c}-1}\left|G_{n, 1}\right|^{2} \rho_{n}+\frac{N_{0}}{E_{S} \operatorname{sinc}^{2}\left(\pi \Delta_{1}\right)}\right]}
\end{aligned}
$$


where

$$
\rho_{n, k}=\operatorname{sinc}^{2}\left(\pi\left(N_{c}(k-1)+n-1+\Delta_{k}\right)\right) / \operatorname{sinc}^{2}\left(\pi \Delta_{1}\right)
$$

is the fraction that gives the MAI contribution from each subcarrier of the $k^{\text {th }}$ interferer, and $\rho_{n}=$ $\operatorname{sinc}^{2}\left(\pi\left(n-1+\Delta_{1}\right)\right) / \operatorname{sinc}^{2}\left(\pi \triangle_{1}\right)$, is the fraction that gives ICI contribution from each subcarrier due to CFO.

Spectral efficiency of the system depends on the SINR in (8), and can be evaluated by using the well known Shannon capacity expression

$$
\mathrm{C}=\mathbb{E}\left[\log _{2}(1+\operatorname{SINR})\right]
$$

where the random variable SINR is given in (13), it consists of $N_{c} \times K$ random variables $G_{0,1}, \ldots, G_{N_{c}-1,1}, \ldots, G_{0, K}, \ldots, G_{N_{c}-1, K}$.

The direct method to compute right hand side of (9) would require $N_{c} \times K$-fold integrations, making it very complex. Gaussian approximation of the interference in the denominator in (8) is incorrect due to correlation between the numerator and the denominator. Moreover, it would still require finding $K$ probability distribution functions of the summations $\sum_{n=0}^{N_{c}-1}\left|G_{n, k}\right|^{2}$, where $k \in[0, K]$. Furthermore, the summation $\sum_{n=0}^{N_{c}-1}\left|G_{n, k}\right|^{2}$ can not be considered as chi square distributed due to correlation between $G_{n, k}$ 's. In the following, we find an accurate yet simpler solution to (9).

\section{Spectral Efficiency Analysis}

In this section, we derive a new lower bound for the spectral efficiency expression in (9), rewritten as

$$
\begin{aligned}
\mathrm{C}=\mathbb{E} & {\left[\operatorname { l o g } _ { 2 } \left(1+\left|G_{1,1}\right|^{2}\left(\sum_{k=2}^{K} \sum_{n=0}^{N_{c}-1}\left|G_{n, k}\right|^{2} \rho_{n, k}\right.\right.\right.} \\
& \left.\left.\left.+\sum_{n=0, n \neq 1}^{N_{c}-1}\left|G_{n, 1}\right|^{2} \rho_{n}+\frac{N_{0}}{E_{S} \operatorname{sinc}^{2}\left(\pi \triangle_{1}\right)}\right)^{-1}\right)\right] .
\end{aligned}
$$

Let $\hat{\rho}_{n, k}=\mathbb{E}\left[\rho_{n, k}\right]$, then Jensen inequality asserts that

$$
\begin{aligned}
\mathrm{C} \geq \mathbb{E} & {\left[\operatorname { l o g } _ { 2 } \left(1+\left|G_{1,1}\right|^{2}\left(\sum_{k=2}^{K} \sum_{n=0}^{N_{c}-1}\left|G_{n, k}\right|^{2} \hat{\rho}_{n, k}\right.\right.\right.} \\
& \left.\left.\left.+\sum_{n=0, n \neq 1}^{N_{c}-1}\left|G_{n, 1}\right|^{2} \rho_{n}+\frac{N_{0}}{E_{S} \operatorname{sinc}^{2}\left(\pi \triangle_{1}\right)}\right)^{-1}\right)\right] .
\end{aligned}
$$

Jensen inequality is applicable because the function is of type $y=\log _{2}(1+a /(x+b))$, and $y$ is always convex $\left(\partial^{2} y / \partial x^{2} \geq 0\right)$ for non-negative $a, b$ and $x$.

Now, we can rewrite (11) in a more desirable form using [22, Lemma 1] to get (14), shown in Appendix.
When $n=1, \rho_{n}=\Delta_{1}^{2} /\left(1-1+\Delta_{1}\right)^{2}=1$, therefore, $\sum_{n=0, n \neq 1}^{N_{c}-1}\left|G_{n, 1}\right|^{2} \rho_{n}+\left|G_{1,1}\right|^{2}$ can be written simply as $\sum_{n=0}^{N_{c}-1}\left|G_{n, 1}\right|^{2} \rho_{n}$, hence (14) can be simplified as in (15), both shown in Appendix. In (15) the Gaussian quadratic forms $\sum_{n=0}^{N_{c}-1}\left|G_{n, k}\right|^{2}$ appear in the exponent. Therefore, known results for the moment generating function of the Gaussian quadratic forms [23, eq. (4a)] can be applied to obtain explicit expression for the lower bound on the spectral efficiency of OFDM in the presence of CFO as given in (16), shown in Appendix. Where, |.| represents determinant of a matrix, $\mathbf{I}_{N_{c}}$ is the $N_{c} \times N_{c}$ identity matrix, whereas $\Lambda$ and $\Lambda_{k}^{1}$ are $N_{c} \times N_{c}$ matrices where a typical element is given by $\Lambda_{i j}=\mathbb{E}\left[G_{i, 1} G_{j, 1}\right] \sqrt{\rho_{i} \rho_{j}}$ and $\Lambda_{k}^{1}(i, j)=\mathbb{E}\left[G_{i, 2} G_{j, 2}^{*} \sqrt{\hat{\rho}_{i, k} \hat{\rho}_{j, k}}\right]$, respectively.

Equation (16) is the desired expression for spectral efficiency with CFO. It is worth mentioning that (16) requires a single integration as compared to $N_{c} \times K$ integrations in the direct method.

\section{Numerical Results}

In this section, we present some numerical and simulation results to show tightness of the spectral efficiency bound. MATLAB was used to simulate and generate all numerical results. Table 1 gives a list of parameter values used in simulation.

In Fig. 1, spectral efficiency using (16) is plotted against $\Delta$, the simulation result was generated using (10) with $10^{6}$ iterations. We used $E_{s} / N_{0}$ of $0 \mathrm{~dB}$ and $10 \mathrm{~dB}$ and the results are shown for a fully loaded system. A $312.5 \mathrm{kHz}$ frequency separation $(1 / T)$ between neighboring subcarriers (IEEE 802.11a) and channel delay spread $(\sigma)$ of $0.1 \mu$ s is used. It can be clearly seen that our analysis provides a tight lower bound. Numerical values of the analysis and simulation results are also shown in Tab. 2 , for $E_{S} / N_{0}$ of $10 \mathrm{~dB}$. The results clearly show the tightness of the lower bound found by our analysis.

In Fig. 2, spectral efficiency degradation due to $\mathrm{CFO}$ is shown for both OFDMA and MC-CDMA at partial loading condition. MC-CDMA shows more resilience to $\mathrm{CFO}$ than OFDMA. A CFO of $25 \%(\Delta=0.25)$ results in a spectral efficiency drop of $23 \%$ (from 0.088 to $0.068 \mathrm{bits} / \mathrm{sec} / \mathrm{Hz}$ ) for OFDMA while for MC-CDMA it only drops $8 \%$ (from 0.096 to $0.088 \mathrm{bits} / \mathrm{sec} / \mathrm{Hz}$ ). This is due to the diversity gain offered by MC-CDMA, which is not present in OFDMA. The spectral efficiency for both MC-CDMA and OFDMA drops to zero when the normalized CFO reaches one as expected, since subcarriers start overlapping.

In Fig. 3, spectral efficiency versus $E_{S} / N_{0}$ is shown for OFDMA and MC-CDMA. The curves are drawn for $\Delta$ values of 0.1 and 0.2 , the spectral efficiency increases with $E_{s} / N_{0}$ as expected and the decrease in spectral efficiency with increasing CFO is also evident. Moreover, the spectral efficiency sharply increases in the range of $0 \mathrm{~dB}$ to $20 \mathrm{~dB}$, 


\begin{tabular}{|c|c|}
\hline Number of Subcarriers $\left(N_{c}\right)$ & 128 \\
\hline Channel delay spread $(\sigma)$ & $1 \mu \mathrm{s}$ \\
\hline $\begin{array}{c}\text { Frequency separation between } \\
\text { adjacent subcarriers }\left(f_{i}-f_{j}\right)\end{array}$ & $312.5 \times 10^{3} \mathrm{~Hz}$ \\
\hline Signal to noise ratio $\left(E_{S} / N_{0}\right)$ & $10 \mathrm{~dB}$ \\
\hline
\end{tabular}

Tab. 1. Simulation parameter values.

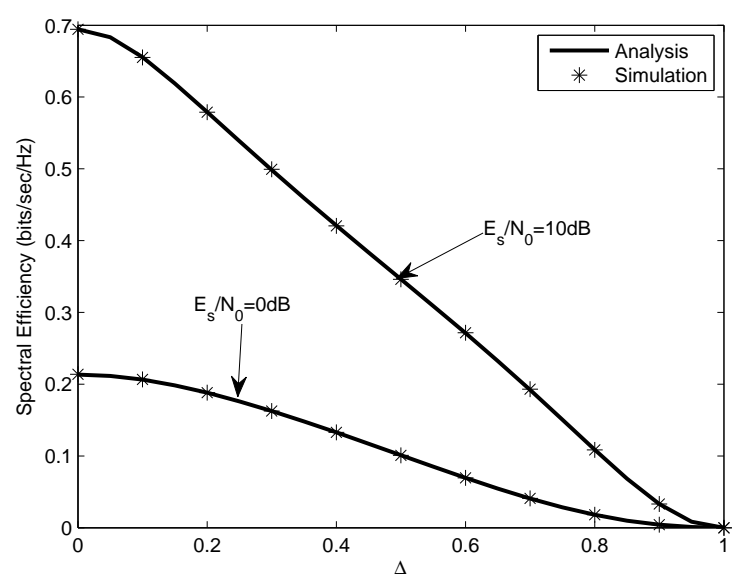

Fig. 1. Spectral efficiency bound versus $\Delta$ of OFDM for some $E_{s} / N_{0}$ values.

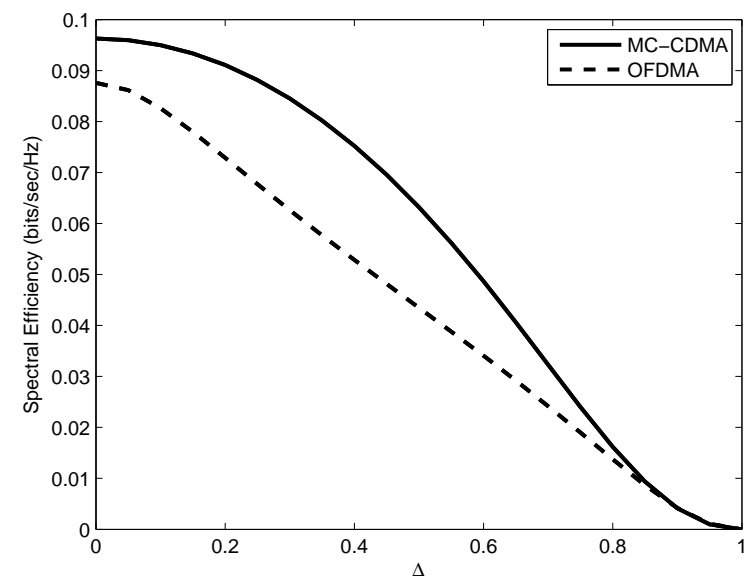

Fig. 2. Degradation in spectral efficiency due to $\Delta$ for OFDM and MC-CDMA.

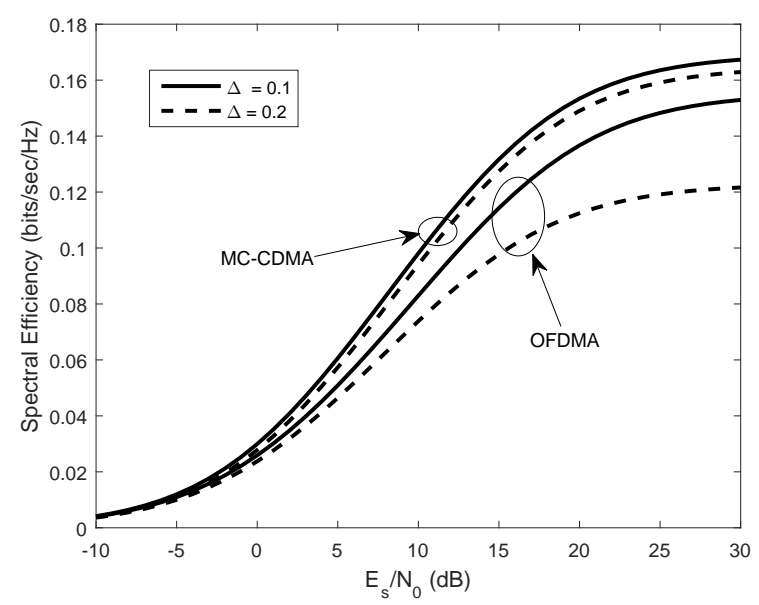

Fig. 3. Spectral efficiency versus $E_{S} / N_{0}$ for $\Delta$ of 0.1 and 0.2 .

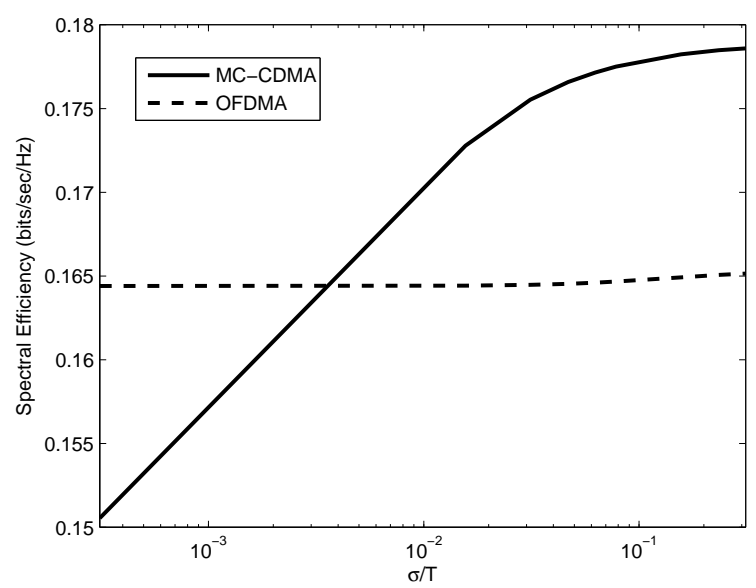

Fig. 4. Spectral efficiency versus $\sigma / T$ for $\Delta=0.2$.

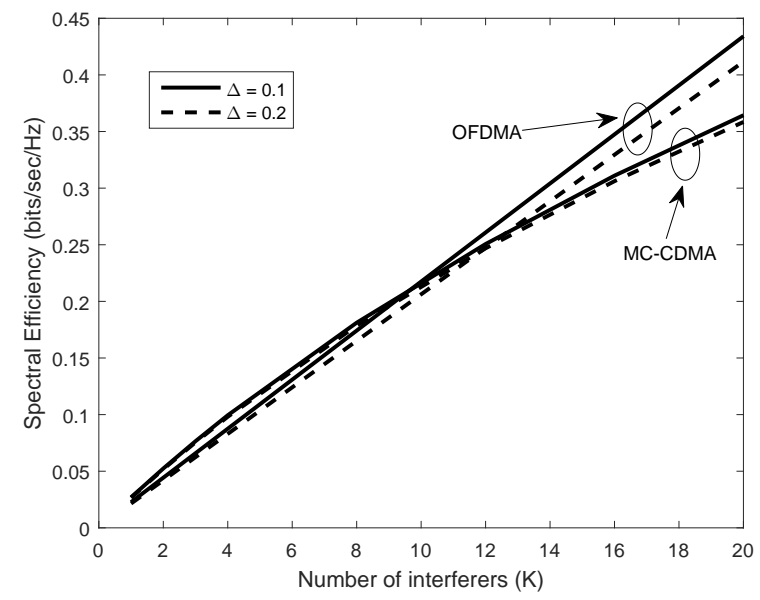

Fig. 5. Spectral efficiency versus $K$ for some $\Delta$ values.

with marginal increase after $10 \mathrm{~dB}$. The degradation effect of CFO is more severe for OFDMA than MC-CDMA, due to, no diversity gain in OFDMA. Furthermore, this degradation is more pronounced at higher $E_{S} / N_{0}$ values as compared to the lower values.

Figure 4 shows the degradation in spectral efficiency against $\sigma$ normalized to $T$. In case of MC-CDMA, larger values of normalized $\sigma$ give better spectral efficiency. In case of OFDMA, no change is observed. This is because, the correlation between subcarriers decays faster when the normalized $\sigma$ is increased, and lower correlation between subcarriers increases the diversity gain of MC-CDMA. Since, OFDMA does not take advantage of lower correlation to offer diversity, hence it's spectral efficiency does not increase.

Figure 5 shows the spectral efficiency versus total number of users/interferers $(K)$ for $\Delta$ of 0.1 and 0.2 . The results are only shown for a maximum $K$ value of 20 , as that is the interesting part of the result. When $K>20$, the spectral efficiency follows the same trend. The spectral efficiency of OFDMA and MC-CDMA monotonically increases with $K$, since the channel utilization is increasing. In case of OFDMA, this increase is almost linear because OFDMA 


\begin{tabular}{|c|c|c|c|c|c|c|c|}
\hline Normalized CFO between transmitter and receiver $(\Delta)$ & 0 & 0.1 & 0.2 & 0.3 & 0.5 & 0.7 & 0.9 \\
\hline \hline Spectral efficiency found by simulation $[\mathrm{bits} / \mathrm{sec} / \mathrm{Hz}]$ & 0.6944 & 0.6562 & 0.5791 & 0.4988 & 0.3465 & 0.1929 & 0.0332 \\
\hline Spectral efficiency found by analysis $[\mathrm{bits} / \mathrm{sec} / \mathrm{Hz}]$ & 0.6944 & 0.6555 & 0.5791 & 0.4983 & 0.3463 & 0.1921 & 0.0330 \\
\hline
\end{tabular}

Tab. 2. Comparison of simulation and analysis results.

users offer little interference to each other. While, in case of MC-CDMA, this increase is more pronounced when the number of users is small but the rate of increase reduces as the number of users increases due to MAI. Comparing the two curves, it is clear that MC-CDMA has higher spectral efficiency than OFDMA at low load condition due to diversity gain offered by MC-CDMA. On the other hand, OFDMA gives higher spectral efficiency than MC-CDMA at high load condition due to smaller interference.

\section{Conclusion}

In this paper, we have presented a new expression for the lower bound of spectral efficiency of OFDMA transmission in the presence of asynchronous users and CFO. Numerical results show that the bound is indeed tight and is a close approximation of the spectral efficiency. Using the spectral efficiency bound we compare the performance of OFDMA and MC-CDMA at several load, SNR, CFO and delay spread conditions. It is clearly evident that MC-CDMA is more resilient to $\mathrm{CFO}$ and takes full advantage of low correlation among subcarriers to offer diversity. However, due to its inherent nature, users generate higher interference to each other than OFDMA. Therefore, at high load condition, OFDMA offers higher spectral efficiency than MC-CDMA. High load condition is normally experienced at peak hours and in dense urban areas.

\section{References}

[1] YEE, N., LINNARTZ, J., FETTWEIS, G. Multicarrier CDMA in indoor wireless radio networks. In Proceedings of the 4th IEEE International Symposium on Personal, Indoor and Mobile Radio Communications (PIMRC). Yokohama (Japan), 1993, p. 109-113.

[2] YEE, N., LINNARTZ, J. P., FETTWEIS, G. Multi-Carrier CDMA in indoor wireless radio networks. IEICE Transactions on Communications, 1994, vol. 77, no. 7, p. 900-904.

[3] DASILVA, V., SOUSA, E. Performance of orthogonal CDMA codes for quasi-synchronous communication systems. In Proceedings of the 2nd IEEE International Conference on Universal Personal Communications. Ottawa (Canada), 1993, vol. 2, p. 995-999. DOI: 10.1109/ICUPC.1993.528528

[4] VANDENDORPE, L. Multitone direct sequence CDMA system in an indoor wireless environment. In Proceedings of the IEEE First Symposium of Communications and Vehicular Technology in the Benelux. Delft (Nederland), 1993, p. 1-8.

[5] CAO, Z., TURELI, U., YAO, Y.-D. Deterministic multiuser carrierfrequency offset estimation for interleaved OFDMA uplink. IEEE Transactions on Communications, 2004, vol. 52, no. 9, p. 1585-1594. DOI: 10.1109/TCOMM.2004.833183
[6] TOMBA, L., KRZYMIEN, W., et al. Sensitivity of the MCCDMA access scheme to carrier phase noise and frequency offset. IEEE Transactions on Vehicular Technology, 1999, vol. 48, no. 5, p. 1657-1665. DOI: 10.1109/25.790546

[7] LIU, Y., TAN, Z. Carrier frequency offset estimation for OFDM systems using repetitive patterns. Radioengineering, 2012, vol. 21, p. 823-830. ISSN: $1210-2512$

[8] KALWAR, S., UMRANI, F. A., MAGARINI, M. Feedback method for estimation and compensation of carrier frequency offset in LTE uplink. In Proceedings of the IEEE International Conference on Computer, Information and Telecommunication Systems (CITS). Kunming (China), 2016, p. 1-4. DOI: 10.1109/CITS.2016.7546402

[9] STANKOVIC, V. Iterative frequency domain maximum likelihood OFDM carrier frequency offset estimation. Wireless Personal Communications, 2016, vol. 91, no. 2, p. 1-13. DOI: 10.1007/s11277016-3508-1

[10] AHMED, J., HAMDI, K. Spectral efficiency of asynchronous MCCDMA with frequency offset over correlated fading. IEEE Transactions on Vehicular Technology, 2013, vol. 62, p. 3423-3429. DOI: 10.1109/TVT.2013.2253339

[11] AHMED, J., HAMDI, K. Spectral efficiency degradation of multicarrier CDMA due to carrier frequency offset. In Proceedings of the 2011 IEEE International Conference on Communications (ICC). Kyoto (Japan), 2011, p. 1-5. DOI: 10.1109/icc.2011.5963490

[12] TONELLO, A., LAURENTI, N., PUPOLIN, S. Analysis of the uplink of an asynchronous multi-user DMT OFDMA system impaired by time offsets, frequency offsets, and multi-path fading. In Proceedings of the Vehicular Technology Conference. 2000, vol. 3, p. 1094-1099. DOI: 10.1109/VETECF.2000.886275

[13] PARK, M., KO, K., PARK, B., et al. Effects of asynchronous MAI on average SEP performance of OFDMA uplink systems over frequencyselective rayleigh fading channels. IEEE Transactions on Communications, 2010, vol. 58, p. 586-599. DOI: 10.1109/TCOMM.2010.02.050324

[14] WANG, X., TJHUNG, T., WU, Y., et al. SER performance evaluation and optimization of OFDM system with residual frequency and timing offsets from imperfect synchronization. IEEE Transactions on Broadcasting, 2003, vol. 49, no. 2, p. 170-177. DOI: 10.1109/TGRS.2003.810271

[15] RUGINI, L., BANELLI, P. BER of OFDM systems impaired by carrier frequency offset in multipath fading channels. IEEE Transactions on Wireless Communications, 2005, vol. 4, p. 2279-2288. DOI: 10.1109/TWC.2005.853884

[16] DU, Z., CHENG, J., BEAULIEU, N. Accurate error-rate performance analysis of OFDM on frequency-selective Nakagami-m fading channels. IEEE Transactions on Communications, 2006, vol. 54, no. 2, p. 319-328. DOI: 10.1109/TCOMM.2005.863729

[17] KELLER, T., HANZO, L. Adaptive multicarrier modulation: A convenient framework for timefrequency processing in wireless communications. Proceedings of the IEEE, 2000, vol. 88, no. 5, p. 611-640. DOI: $10.1109 / 5.849157$

[18] ALMRADI, A., HAMDI, K. Spectral efficiency of OFDM systems with random residual CFO. IEEE Transactions on Communications, 2015, vol. 63, no. 7, p. 2580-2590. DOI: 10.1109/TCOMM.2015.2443103 
[19] HAMDI, K. Average capacity analysis of OFDM with frequency offset in Rician fading. In Proceedings of the IEEE Global Telecommunications Conference (GLOBECOM). 2007, p. 1678-1682. DOI: 10.1109/GLOCOM.2007.323

[20] MATHECKEN, P., RIIHONEN, T., WERNER, S., et al. Average capacity of rayleigh-fading OFDM link with Wiener phase noise and frequency offset. In Proceedings of the IEEE 23rd International Symposium on Personal, Indoor and Mobile Radio Communications (PIMRC). 2012, p. 2353-2358. DOI: 10.1109/PIMRC.2012.6362750

[21] W. C. JAKES. Microwave Mobile Communications. 1st ed. IEEE Press, 1974. ISBN: 97804705452

[22] HAMDI, K. A useful lemma for capacity analysis of fading interference channels. IEEE Transactions on Communications, 2010, vol. 58, no. 2, p. 411-416. DOI: 10.1109/TCOMM.2010.02.080117
[23] TURIN, G. L. The characteristic function of Hermitian quadratic forms in complex normal variables. Biometrika, 1960, p. 199-201. DOI: 10.1093/biomet/47.1-2.199

\section{About the Author...}

Junaid AHMED is Assistant Professor at COMSATS Institute of Information Technology, Islamabad, Pakistan. He graduated from Middle East Technical University in Ankara, Turkey in 1997. His MSc degree is from Oklahoma State University, Stillwater, Oklahoma, USA and PhD. degree from The University of Manchester, UK in 2012. His research interests include wireless communications and networks, and $5 \mathrm{G}$ cellular communications.

\section{Appendix:}

$$
\begin{array}{r}
\operatorname{Var}\left[Z \mid G_{0,1}, \ldots, G_{N_{c}-1, K}, \Delta_{2}, \ldots, \Delta_{K}\right]=\frac{E_{s}}{T}\left(\left|G_{1,1}\right|^{2} \sum_{k=2}^{K} \sum_{n_{k}=0}^{N_{c}-1}\left|G_{n, k}\right|^{2} \operatorname{sinc}^{2}\left(\pi\left(N_{c}(k-1)+n-1+\Delta_{k}\right)\right)+\left|G_{1,1}\right|^{2}\right. \\
\left.\sum_{n_{1}=0, n_{1} \neq 1}^{N_{c}-1}\left|G_{n, 1}\right|^{2} \operatorname{sinc}^{2}\left(\pi\left(n_{1}-1+\Delta_{1}\right)\right)\right)+\frac{N_{0}}{T}\left|G_{1,1}\right|^{2} .
\end{array}
$$

$$
\begin{aligned}
& \operatorname{SINR}=\frac{E_{S}}{T}\left|G_{1,1}\right|^{4} \operatorname{sinc}^{2}\left(\pi \triangle_{1}\right)\left[\frac { E _ { S } } { T } \left(\left|G_{1,1}\right|^{2} \sum_{k=2}^{K} \sum_{n_{k}=0}^{N_{c}-1}\left|G_{n, k}\right|^{2} \operatorname{sinc}^{2}\left(\pi\left(N_{c}(k-1)+n-1+\Delta_{k}\right)\right)\right.\right. \\
& \left.\left.+\left|G_{1,1}\right|^{2} \sum_{n_{1}=0, n_{1} \neq 1}^{N_{c}-1}\left|G_{n, 1}\right|^{2} \operatorname{sinc}^{2}\left(\pi\left(n_{1}-1+\triangle_{1}\right)\right)\right)+\frac{N_{0}}{T}\left|G_{1,1}\right|^{2}\right]^{-1} .
\end{aligned}
$$

$$
\begin{aligned}
\mathrm{C} \geq \log _{2} \mathrm{e} \int_{0}^{\infty} \frac{1}{z}(\mathbb{E} & {\left[\exp \left(-z\left(\sum_{k=2}^{K} \sum_{n=0}^{N_{c}-1}\left|G_{n, k}\right|^{2} \hat{\rho}_{n, k}+\sum_{n=0, n \neq 1}^{N_{c}-1}\left|G_{n, 1}\right|^{2} \rho_{n}\right)\right)\right] } \\
-\mathbb{E} & {\left[\exp \left(-z\left(\sum_{k=2}^{K} \sum_{n=0}^{N_{c}-1}\left|G_{n, k}\right|^{2} \hat{\rho}_{n, k}+\sum_{n=0, n \neq 1}^{N_{c}-1}\left|G_{n, 1}\right|^{2} \rho_{n}+\left|G_{1,1}\right|^{2}\right)\right)\right] \exp \left(-\frac{z}{\operatorname{sinc}^{2}\left(\pi \Delta_{1}\right)} \frac{N_{0}}{E_{s}}\right) \mathrm{d} z }
\end{aligned}
$$

$$
\begin{gathered}
\mathrm{C} \geq \log _{2} \mathrm{e} \int_{0}^{\infty} \frac{1}{z}\left(\mathbb{E}\left[\exp \left(-z\left(\sum_{k=2}^{K} \sum_{n=0}^{N_{c}-1}\left|G_{n, k}\right|^{2} \hat{\rho}_{n, k}+\sum_{n=0, n \neq 1}^{N_{c}-1}\left|G_{n, 1}\right|^{2} \rho_{n}\right)\right)\right]\right. \\
\quad-\mathbb{E}\left[\exp \left(-z\left(\sum_{k=2}^{K} \sum_{n=0}^{N_{c}-1}\left|G_{n, k}\right|^{2} \hat{\rho}_{n, k}+\sum_{n=0}^{N_{c}-1}\left|G_{n, 1}\right|^{2} \rho_{n}\right)\right)\right] \exp \left(-\frac{z}{\operatorname{sinc}^{2}\left(\pi \Delta_{1}\right)} \frac{N_{0}}{E_{s}}\right) \mathrm{d} z \\
\mathrm{C} \geq \log _{2} \mathrm{e} \int_{0}^{\infty} \frac{1}{z} \prod_{k=2}^{K}\left(\frac{1}{\left|\mathbf{I}_{N_{C}}+z \Lambda_{k}^{1}\right|}\right)\left(\frac{1}{\left|\mathbf{I}_{N_{c}-1}+z \Lambda\right|}-\frac{1}{\left|\mathbf{I}_{N_{c}}+z \Lambda\right|}\right) \exp \left(-\frac{z}{\operatorname{sinc}^{2}\left(\pi \Delta_{1}\right)} \frac{N_{0}}{E_{s}}\right) \mathrm{d} z
\end{gathered}
$$

